\title{
A CHARACTERIZATION OF THE TEMPERED DISTRIBUTIONS SUPPORTED BY A REGULAR CLOSED SET IN THE HEISENBERG GROUP
}

By

\author{
Yasuyuki OKA
}

\begin{abstract}
The aim of this paper is to give a characterization of the tempered distributions supported by a (Whitney's) regular closed set in the Euclidean space and the Heisenberg group by means of the heat kernel method. The heat kernel method, introduced by $\mathrm{T}$. Matsuzawa, is the method to characterize the generalized functions on the Euclidean space by the initial value of the solutions of the heat equation.
\end{abstract}

\section{Introduction}

A regular closed set played an important role in the Whitney extension theorem ([22]) and in the structure theorem of the distributions with support ([18]). The examples of a regular closed set in the Euclidean space are convex compact sets, an upper half plane and so on.

In this article, we consider the tempered distributions supported by a regular closed set in the Euclidean space and in the Heisenberg group, respectively. The Heisenberg group is the most commutative in the non-commutative Lie group.

Our goals are two characterizations as follows. At first, we will give the characterization of the tempered distributions supported by a regular closed set in the Euclidean space by means of the heat kernel method. The heat kernel method, introduced by T. Matsuzawa in [14], is the method to characterize the generalized functions on the Euclidean space by the initial value of the solutions

2010 Mathematics Subject Classification: 46F05; 46F15; 81S30.

Key words and phrases: Tempered distributions in the Heisenberg group, Heat equation, regular closed sets in the Heisenberg group.

Received October 14, 2014.

Revised January 15, 2015. 
of the heat equation. Especially, in [15], T. Matsuzawa showed the heat kernel method for the tempered distributions on "the Euclidean space $\mathbf{R}^{d}$ ". By using Matsuzawa's result, we will get our first assertion.

Secondly, we will give the characterization of the tempered distributions supported by a regular closed set in the Heisenberg group by means of the heat kernel method. To do this, we will give the definition of a regular closed set in the Heisenberg group. A regular closed set in the Heisenberg group include the geodetically convex sets. In the Heisenberg group, the heat kernel method for the tempered distributions was given by J. Kim and M. W. Wong in [12]. By using their result, we will obtain our second assertion. We also have the structure theorem of the tempered distributions supported by a regular closed set in the Heisenberg group (Bros-Epstien-Glaser type theorem) on one's way.

As an application of the characterizations by means of the heat kernel method, we will give the Schwartz kernel theorem for the tempered distributions supported by a regular closed set in the Euclidean space and in the Heisenberg group, respectively. Recently, the Schwartz kernel theorem is adopted by $\mathrm{R}$. Ashino, T. Mandai and A. Morimoto (in [1]) in consideration on BIBO (Bounded-Input Bounded-Output) stability of the continuous linear time shift invariant system on the Euclidean space. As far as the BIBO stability of the continuous linear time shift invariant system, we also refer to [17].

The plan of this paper is as follows: In section 2, we will give the characterization of the tempered distributions supported by a regular closed set $A$ on the Euclidean space by the heat kernel. In 2.1, we introduce the properties of the heat kernel and the heat kernel method for the tempered distributions (Theorem 1) on the Euclidean space. In 2.2, we recall the definition of a regular closed set and define the space $\mathscr{S}(A)$. It is shown that the space $\mathscr{S}\left(\mathbf{R}^{d}\right)$ is dense in the space $\mathscr{S}(A)$. Moreover we introduce the structure theorem for the space $\mathscr{S}(A)^{\prime}$ (the Bros-Epstein-Glaser type theorem), where the space $\mathscr{S}(A)^{\prime}$ is the set of the tempered distributions supported by $A$. In 2.3 , we will show the heat kernel method for the space $\mathscr{S}(A)^{\prime}$ (Our first assertion). In 2.4, as an application of this characterization, we will give the Schwartz kernel theorem for the space $\mathscr{S}(A)^{\prime}$.

In section 3, we will give the characterization of the tempered distributions supported by a regular closed set $A_{\mathbf{H}^{d}}$ in the Heisenberg group by the heat kernel method. In 3.1, we recall the definition of the Heisenberg group, the form of the left invariant vector fields in the Heisenberg group, the distance function called Korányi norm and the definition of the convolution in the Heisenberg group. In 3.2, we give the definition of the rapidly decreasing functions and the tempered 
distributions in the Heisenberg group. In 3.3, we introduce the properties of the heat kernel and the heat kernel method in the Heisenberg group. In 3.4, we define a regular closed set $A_{\mathbf{H}^{d}}$ in the Heisenberg group and the space $\mathscr{S}\left(A_{\mathbf{H}^{d}}\right)$. Then we will show that the space $\mathscr{S}\left(\mathbf{H}^{d}\right)$ is dense in $\mathscr{S}\left(A_{\mathbf{H}^{d}}\right)$. Moreover we will also give the structure theorem for the space $\mathscr{S}\left(A_{\mathbf{H}^{d}}\right)^{\prime}$ (The Bros-Epstein-Glaser type theorem), where the space $\mathscr{S}\left(A_{\mathbf{H}^{d}}\right)^{\prime}$ is the tempered distributions supported by $A_{\mathbf{H}^{d}}$ in the Heisenberg group. In 3.5, we will show the heat kernel method for the space $\mathscr{S}\left(A_{\mathbf{H}^{d}}\right)^{\prime}$ (our second assertion). In 3.6, as an application of this result, we will give the Schwartz kernel theorem for the space $\mathscr{S}\left(A_{\mathbf{H}^{d}}\right)^{\prime}$. Finally, as an appendix, we will show some examples of a regular closed set in the Heisenberg group $\mathbf{H}$.

\section{A Characterization of the Tempered Distributions Supported by a Regular Closed Set in the Euclidean Space by the Heat Kernel Method}

\subsection{The Heat Kernel Method for the Space $\mathscr{S}^{\prime}\left(\mathbf{R}^{d}\right)$}

First of all, we fix some notations. We use a multi-index $\alpha \in \mathbf{Z}_{+}^{d}$, namely, $\alpha=\left(\alpha_{1}, \ldots, \alpha_{d}\right)$, where $\alpha_{i} \in \mathbf{Z}$ and $\alpha_{i} \geq 0$. So, for $x \in \mathbf{R}^{d}, x^{\alpha}=x_{1}^{\alpha_{1}} \cdots x_{d}^{\alpha_{d}}$ and $\partial_{x}^{\alpha}=\partial_{x_{1}}^{\alpha_{1}} \cdots \partial_{x_{d}}^{\alpha_{d}}$, where $\partial_{x_{j}}^{\alpha_{j}}=\left(\partial / \partial x_{j}\right)^{\alpha_{j}}$. Moreover $\Delta=\sum_{j=1}^{d} \partial^{2} / \partial x_{j}^{2}$.

Definition 1. For any $\varphi \in C^{\infty}\left(\mathbf{R}^{d}\right)$, we say $\varphi \in \mathscr{S}\left(\mathbf{R}^{d}\right)$ if the function $\varphi$ satisfies the following condition: For any $N \in \mathbf{Z}_{+}$, we have

$$
\|\varphi\|_{N, \mathbf{R}^{d}}=\sup _{x \in \mathbf{R}^{d},|\beta| \leq N}(1+|x|)^{N}\left|\partial_{x}^{\beta} \varphi(x)\right|<\infty .
$$

Moreover we denote by $\mathscr{S}^{\prime}\left(\mathbf{R}^{d}\right)$ the dual space of the space $\mathscr{S}\left(\mathbf{R}^{d}\right)$. Thus, we say $T \in \mathscr{S}^{\prime}\left(\mathbf{R}^{d}\right)$ if a continuous and linear functional $u$ from $\mathscr{S}\left(\mathbf{R}^{d}\right)$ to $\mathbf{C}$ satisfies the following condition: There exist a constant $C>0$ and $\alpha, \beta \in \mathbf{Z}_{+}^{d}$ such that

$$
|\langle T, \varphi\rangle| \leq C\|\varphi\|_{N, \mathbf{R}^{d}}
$$

for any $\varphi \in \mathscr{S}\left(\mathbf{R}^{d}\right)$. It is called the space of the tempered distributions.

The function $E_{t}(x)$ defined by

$$
E_{t}(x)=\left\{\begin{array}{l}
(4 \pi t)^{-d / 2} e^{-|x|^{2} / 4 t} \quad(t>0) \\
0 \quad(t<0)
\end{array}\right.
$$

is called the heat kernel on $\mathbf{R}^{d}$. Then the heat kernel $E_{t}(x)$ on $\mathbf{R}^{d}$ has the following Proposition 1: 
Proposition 1 ([4]). The following properties hold:

(i) $\int_{\mathbf{R}^{d}} E_{t}(x) d x=1, t>0$,

(ii) There are positive constants $C$ and $a^{\prime}$ such that

$$
\left|\partial_{x}^{\beta} E_{t}(x)\right| \leq C^{|\beta|+1} t^{-(n+|\alpha|) / 2} \beta !^{1 / 2} e^{-a^{\prime}|x|^{2} / 4 t}, \quad t>0,0<a^{\prime}<1,
$$

(iii) $E_{t}(x) \in \mathscr{S}\left(\mathbf{R}_{x}^{d}\right)$.

Proposition 2 ([13]). Let $\varphi \in \mathscr{S}\left(\mathbf{R}^{d}\right)$, Then we have

$$
\left(\varphi * E_{t}\right)(x) \rightarrow \varphi
$$

as $t \rightarrow+0$ in $\mathscr{S}\left(\mathbf{R}^{d}\right)$.

In [15], T. Matsuzawa characterized the tempered distributions on the Euclidean space by the initial value of the solutions of the heat equation as follows:

THEOREM 1 ([15]). For $u \in \mathscr{S}^{\prime}\left(\mathbf{R}^{d}\right)$, we put

$$
U_{t}(x)=\left(u * E_{t}\right)(x)
$$

for $x \in \mathbf{R}^{d}$ and $t>0$. Then the function $U_{t}(x)$ satisfies the following four conditions:

(i) $U_{t}(x) \in C^{\infty}\left(\mathbf{R}^{d} \times(0, \infty)\right)$,

(ii) $\left(\partial / \partial t-\Delta_{x}\right) U_{t}(x)=0, x \in \mathbf{R}^{d}$ and $t>0$,

(iii) for any $\varphi \in \mathscr{S}\left(\mathbf{R}^{d}\right)$,

$$
\langle u, \varphi\rangle=\lim _{t \rightarrow+0} \int_{\mathbf{R}^{d}} U_{t}(x) \varphi(x) d x
$$

and

(iv) there exist $\mu, v>0$ and a constant $C>0$ such that

$$
\left|U_{t}(x)\right| \leq C t^{-\mu}(1+|x|)^{v}, \quad 0<t<1,
$$

for $x \in \mathbf{R}^{d}$.

Conversely every $U_{t}(x) \in C^{\infty}\left(\mathbf{R}^{d} \times(0, \infty)\right)$ satisfying the conditions (ii) and (iv) can be expressed in the form

$$
U_{t}(x)=\left(u * E_{t}\right)(x)
$$

with the unique element $u \in \mathscr{S}^{\prime}\left(\mathbf{R}^{d}\right)$. 
This characterization is called the heat kernel method.

\subsection{The Structure of the Tempered Distributions Supported by Regular Closed Sets on $\mathbf{R}^{d}$}

At first, we recall the definition of a regular closed set.

Definition 2 ([8], [21], [22]). Let $A$ be a closed subset of $\mathbf{R}^{d}$. If there exist $d>0, \omega>0$ and $0<q \leq 1$ such that any $x_{1}$ and $x_{2} \in A$ so that $\left|x_{1}-x_{2}\right| \leq d$ are linked by a curve in $A$ whose length $l$ satisfies $l \leq \omega\left|x_{1}-x_{2}\right|^{q}$, then we call $A a$ regular.

For example, if $A$ is a convex closed set, $\omega=q=1$ and $d=d(A)$ and if $A$ is a closure of the upper half-plane, $\omega=q=1$ and $d=\infty$. Of course, a closure of the first quadrant (a proper convex cone) and the light cone are also a regular closed set.

We define the space $\mathscr{S}(A)$ as follows:

Definition 3. Let $A$ be a regular closed set on $\mathbf{R}^{d}$. For any $\varphi \in C^{\infty}\left(\mathbf{R}^{d}\right)$, we say $\varphi \in \mathscr{S}(A)$ if the function $\varphi$ satisfies the following condition: For any $N \in \mathbf{Z}_{+}$, we have

$$
\|\varphi\|_{N, A}=\sup _{x \in A,|\beta| \leq N}(1+|x|)^{N}\left|\partial^{\beta} \varphi(x)\right|<\infty .
$$

The following relationship between the spaces $\mathscr{S}\left(\mathbf{R}^{d}\right)$ and $\mathscr{S}(A)$ holds:

Proposition 3. The space $\mathscr{S}\left(\mathbf{R}^{d}\right)$ is dense in the space $\mathscr{S}(A)$.

Proof. It is enough that the space $\mathscr{D}\left(\mathbf{R}^{d}\right)$ is dense in the space $\mathscr{S}(A)$. We choose $\chi_{j} \in \mathscr{D}\left(\mathbf{R}^{d}\right)$ as follows:

$$
\chi_{j}(x)= \begin{cases}1, & |x| \leq j \\ 0, & |x| \geq 2 j\end{cases}
$$

for $j=1,2, \ldots$ Let $f$ be in $\mathscr{S}(A)$. If we set $\psi_{j}=f \chi_{j}$, the function $\psi_{j}$ is in $\mathscr{D}\left(\mathbf{R}^{d}\right)$. On the other hand, we have

$$
\partial^{\beta}\left\{\left(1-\chi_{j}\right) f\right\}=\sum_{\gamma \leq \beta}\left(\begin{array}{l}
\beta \\
\gamma
\end{array}\right) \partial^{\gamma}\left(1-\chi_{j}\right) \partial^{\beta-\gamma} f
$$


For $|x| \leq j$, we can see

$$
\partial^{\beta}\left(1-\chi_{j}\right)=0
$$

If the set $A$ is compact, then by (2.1), we can see that

$$
\lim _{j \rightarrow \infty}\left\|f-\psi_{j}\right\|_{N, A}=0 .
$$

On the other hand, for unbounded sets $A$, we obtain the following estimate: For a sufficient large $j$, we have

$$
\begin{aligned}
\left\|f-\psi_{j}\right\|_{N, A} & =\left\|\left(1-\chi_{j}\right) f\right\|_{N, A} \\
& \leq \sup _{x \in A \backslash(\{|x| \leq j\} \cap A),|\beta| \leq N}(1+|x|)^{N}\left|\partial^{\beta}\left\{\left(1-\chi_{j}\right) f\right\}\right| \\
& \leq \sup _{x \in A \backslash(\{|x| \leq j\} \cap A),|\beta| \leq N}(1+|x|)^{N}\left(\sum_{\delta \leq \beta}\left(\begin{array}{c}
\beta \\
\delta
\end{array}\right)\left|\partial^{\delta}\left(1-\chi_{j}\right)\right|\left|\partial^{\beta-\delta} f\right|\right) .
\end{aligned}
$$

Since $f \in \mathscr{S}(A)$, we have for $\delta \leq \beta$,

$$
\sup _{x \in A \backslash(\{|x| \leq j\} \cap A),|\beta| \leq N}(1+|x|)^{N}\left|\partial^{\beta-\delta} f\right| \rightarrow 0
$$

as $j \rightarrow+\infty$. By (2.2), for any $f \in \mathscr{S}(A)$, there exists the sequence $\left\{\psi_{j}\right\}_{j \in \mathbf{N}} \subset$ $\mathscr{D}\left(\mathbf{H}^{d}\right)$ such that

$$
\lim _{j \rightarrow+\infty}\left\|f-\psi_{j}\right\|_{N, A}=0 .
$$

Therefore we can see that the space $\mathscr{D}\left(\mathbf{H}^{d}\right)$ is dense in the space $\mathscr{S}(A)$.

Definition 4. We denote by $\mathscr{S}(A)^{\prime}$ the dual space of the space $\mathscr{S}(A)$. Thus, $u \in \mathscr{S}(A)^{\prime}$ if and only if $u$ is a linear functional from $\mathscr{S}(A)$ to $\mathbf{C}$ and satisfies the following condition: There exist $N \in \mathbf{Z}_{+}$and a positive constant $C$ such that

$$
|\langle u, \varphi\rangle| \leq C\|\varphi\|_{N, A}
$$

for any $\varphi \in \mathscr{S}(A)$.

Here we denote by $\mathscr{S}_{A}^{\prime}$ the space of the tempered distributions $u$ on $\mathbf{R}^{d}$ satisfying the following condition: For any $\varphi \in \mathscr{S}\left(\mathbf{R}^{d}\right)$, there exists a constant $C>0$ such that

$$
|\langle u, \varphi\rangle| \leq C\|\varphi\|_{N, A}
$$


for some $N \in \mathbf{Z}_{+}$. Thus the space $\mathscr{S}_{A}^{\prime}$ means the space of the tempered distributions with supported by $A$ in $\mathbf{R}^{d}$. Then by Proposition 3, (2.3) means that $u$ has continuous on $\mathscr{S}^{\prime}\left(\mathbf{R}^{d}\right)$ with respect to the relative topology from $\mathscr{S}(A)$. Hence $u$ has a unique linear continuous extension $u_{A}$ on $\mathscr{S}(A)$. This means that any tempered distributions supported by $A$ on $\mathbf{R}^{d}$ can be identified with an element of $\mathscr{S}(A)^{\prime}$. Thus, we identify the space $\mathscr{S}_{A}^{\prime}$ with the space $\mathscr{S}(A)^{\prime}$.

Concerning on the tempered distributions supported by a regular closed set, the following result is known:

Proposition 4 ([8], [21]). Let $A$ be a regular closed set. If $f \in \mathscr{S}(A)^{\prime}$, then there exist the tempered measures supported on $A, \mu_{\beta}(|\beta| \leq N)$, such that supp $\mu_{\beta} \subset A$ and

$$
f=\sum_{|\beta| \leq N} \partial^{\beta} \mu_{\beta},
$$

where the tempered measure $\mu$ supported by $A$ means that there exists $N \in \mathbf{Z}_{+}$so that

$$
\int_{A}|d \mu|(x) /(1+|x|)^{N}<\infty
$$

\subsection{The Heat Kernel Method for the Space of the Tempered Distributions Supported by a Regular Closed Set on $\mathbf{R}^{d}$}

We denote by $\mathscr{S}(A)^{\prime}$ the space of the tempered distributions supported by a regular closed set $A$ in $\mathbf{R}^{d}$. Then we obtain the following characterization:

THeOREM 2. Let $A$ be a regular closed set on $\mathbf{R}^{d}$. For any $u$ in $\mathscr{S}(A)^{\prime}$, let $U_{t}(x)=\left\langle u, E_{t}(x-\cdot)\right\rangle$. Then $U_{t}(x)$ satisfies the following conditions:

(i) $U_{t}(x) \in C^{\infty}\left(\mathbf{R}^{d} \times(0, \infty)\right)$,

(ii) $\left(\partial / \partial t-\Delta_{x}\right) U_{t}(x)=0, x \in \mathbf{R}^{d}$ and $t>0$,

(iii) for any $\varphi \in \mathscr{D}\left(\mathbf{R}^{d}\right)$,

and

$$
\langle u, \varphi\rangle=\lim _{t \rightarrow+0} \int_{\mathbf{R}^{d}} U_{t}(x) \varphi(x) d x
$$

(iv) there exist $\mu, N \in \mathbf{Z}_{+}$and constants $C>0$ and $a^{\prime}$ such that

$$
\left|U_{t}(x)\right| \leq C t^{-\mu}(1+|x|)^{N} e^{-a^{\prime} d(x, A)^{2} / 8 t}, \quad 0<t<1,0<a^{\prime}<1
$$

for $x \in \mathbf{R}^{d}$, where $d(x, A)=\inf _{x^{\prime} \in A}\left|x-x^{\prime}\right|$. 
Conversely every $U_{t}(x) \in C^{\infty}\left(\mathbf{R}^{d} \times(0, \infty)\right)$ satisfying the conditions (ii) and (iv) can be expressed in the form

$$
U_{t}(x)=\left(u * E_{t}\right)(x)
$$

with the unique element $u \in \mathscr{S}(A)^{\prime}$.

Proof. Let $u \in \mathscr{S}(A)^{\prime}$ and $U_{t}(x)=\left\langle u, E_{t}(x-\cdot)\right\rangle$. Then by Proposition 1 and Proposition 4, there are $N \in \mathbf{Z}_{+}, C, C^{\prime}>0$ and $0<a^{\prime}<1$ such that

$$
\begin{aligned}
\left|U_{t}(x)\right| & =\left|\left\langle u, E_{t}(x-\cdot)\right\rangle\right| \\
& =\left|\sum_{|\beta| \leq N}(-1)^{|\beta|} \int_{A}(1+|y|)^{N} \partial_{y}^{\beta} E_{t}(x-y) d y\right| \\
& \leq C t^{-(d+N) / 2}(1+|x|)^{N} \sum_{|\beta| \leq N} \int_{A}(1+|x-y|)^{N} e^{-a^{\prime}|x-y|^{2} / 4 t} d y \\
& \leq C^{\prime} t^{-(d+N) / 2}(1+|x|)^{N} e^{-a^{\prime} d(x, A)^{2} / 8 t}
\end{aligned}
$$

for $0<t<1$. Conversely, if any $U_{t}(x) \in C^{\infty}\left(\mathbf{R}^{d}\right)$ satisfies the condition (ii) and (iv), then by Theorem 1 , there exists $u \in \mathscr{S}^{\prime}\left(\mathbf{R}^{d}\right)$ such that

$$
U_{t}(x)=\left\langle u, E_{t}(x-\cdot)\right\rangle .
$$

Let $\varphi \in \mathscr{D}\left(\mathbf{R}^{d}\right)$ and $K=\operatorname{supp} \varphi \subset \mathbf{R}^{d} \backslash A$. Then there are a constant $C_{K, v}>0$ and $\mu \geq 0$ such that

$$
\begin{aligned}
\left|\int_{K} U_{t}(x) \varphi(x) d x\right| & \leq \int_{K}\left|U_{t}(x)\right||\varphi(x)| d x \\
& \leq C_{K, v} t^{-\mu} e^{-a^{\prime} d(K, A)^{2} / 8 t} \\
& \rightarrow 0
\end{aligned}
$$

as $t \rightarrow+0$, where $d(K, A)=\inf _{x \in K} d(x, A)$. Hence we obtain

$$
\lim _{t \rightarrow+0} \int_{K} U_{t}(x) \varphi(x) d x=0 .
$$

On the other hand, by Theorem 1, we have

$$
\lim _{t \rightarrow+0} U_{t}(x)=u(x) \quad \text { in } \mathscr{S}^{\prime}\left(\mathbf{R}^{d}\right) .
$$

Therefore we can see $\operatorname{supp} u \subset A$. 


\subsection{The Schwartz Kernel Theorem for the Space $\mathscr{S}(A)^{\prime}$}

As an application of Theorem 2, we show the Schwartz kernel theorem for the space $\mathscr{S}(A)^{\prime}$ as follows:

THEOREM 3. Let the sets $A_{1}$ and $A_{2}$ be regular closed sets in $\mathbf{R}^{d_{1}}$ and $\mathbf{R}^{d_{2}}$, respectively and $k$ be a continuous linear operator from $\mathscr{S}\left(A_{2}\right)$ to $\mathscr{S}\left(A_{1}\right)^{\prime}$. Then there exists $T$ in $\mathscr{S}\left(A_{1} \times A_{2}\right)^{\prime}$ such that

$$
\langle k \psi, \varphi\rangle=\langle T, \varphi \otimes \psi\rangle,
$$

where $\varphi$ is in $\mathscr{S}\left(A_{1}\right)$ and $\psi$ is in $\mathscr{S}\left(A_{2}\right)$.

Proof. Since $k$ is continuous, the bilinear form $\mathbf{B}$ on $\mathscr{S}\left(A_{1}\right) \times \mathscr{S}\left(A_{2}\right)$,

$$
\mathbf{B}(\varphi, \psi)=\langle k \psi, \varphi\rangle, \quad \varphi \in \mathscr{S}\left(A_{1}\right), \psi \in \mathscr{S}\left(A_{2}\right)
$$

is separately continuous. Since $\mathscr{S}\left(A_{1}\right)$ and $\mathscr{S}\left(A_{2}\right)$ are the Fréchet space respectively, $\mathbf{B}$ is continuous. Hence we can see that there exist a positive constant $C$ and $N_{1}, N_{2} \in \mathbf{Z}_{+}$such that

$$
|\langle k \psi, \varphi\rangle| \leq C\|\varphi\|_{N_{1}, A_{1}}\|\psi\|_{N_{2}, A_{2}} .
$$

We define $R_{t}\left(x_{1}, x_{2}\right)$ by

$$
R_{t}\left(x_{1}, x_{2}\right)=\left\langle k E_{t}\left(x_{2}-\cdot\right), E_{t}\left(x_{1}-\cdot\right)\right\rangle
$$

for $\left(x_{1}, x_{2}\right) \in \mathbf{R}^{d_{1}} \times \mathbf{R}^{d_{2}}$ and $t>0$.

Now we will show $R_{t}$ converges in $\mathscr{S}\left(A_{1} \times A_{2}\right)^{\prime}$ as $t \rightarrow+0$. By $(\sharp)$ and Proposition 1, there exist a positive constant $C$ and $\mu, N_{1}, N_{2} \in \mathbf{Z}_{+}$such that

$$
\left|R_{t}\left(x_{1}, x_{2}\right)\right| \leq C t^{-\mu}\left(1+\left|x_{1}\right|\right)^{N_{1}}\left(1+\left|x_{2}\right|\right)^{N_{2}} e^{-a^{\prime} d\left(x_{1}, A_{1}\right)^{2} / 8 t} e^{-a^{\prime} d\left(x_{2}, A_{2}\right)^{2} / 8 t},
$$

for $x_{1} \in \mathbf{R}^{d_{1}}, x_{2} \in \mathbf{R}^{d_{2}}$ and $0<t<1$.

Moreover we obtain

$$
(\partial / \partial t-\Delta) R_{t}\left(x_{1}, x_{2}\right)=0
$$

for $x_{1} \in \mathbf{R}^{d_{1}}, x_{2} \in \mathbf{R}^{d_{2}}$ and $0<t<1$.

Therefore, by Theorem 2, there exists $R_{0} \in \mathscr{S}^{\prime}\left(\mathbf{R}^{d_{1}} \times \mathbf{R}^{d_{2}}\right)$, supp $R_{0} \subset$ $A_{1} \times A_{2}$, such that

$$
R_{0}=\lim _{t \rightarrow+0} R_{t}
$$

in $\mathscr{S}^{\prime}\left(\mathbf{R}^{d_{1}} \times \mathbf{R}^{d_{2}}\right)$. 
Since the Riemann sum of an integral converges in $\mathscr{S}\left(\mathbf{R}^{d_{j}}\right), j=1,2$, by Theorem 1, we have

$$
\begin{aligned}
\left\langle R_{t}, \varphi \otimes \psi\right\rangle & =\iint_{\mathbf{R}^{d_{1}} \times \mathbf{R}^{d_{2}}} R_{t}\left(x_{1}, x_{2}\right) \varphi\left(x_{1}\right) \psi\left(x_{2}\right) d x_{1} d x_{2} \\
& =\left\langle k \int_{\mathbf{R}^{d_{2}}} E_{t}\left(x_{2}-\cdot\right) \psi\left(x_{2}\right) d x_{2}, \int_{\mathbf{R}^{d_{1}}} E_{t}\left(x_{1}-\cdot\right) \varphi\left(x_{1}\right) d x_{1}\right\rangle \\
& =\left\langle k\left[\psi * E_{t}\right], \varphi * E_{t}\right\rangle
\end{aligned}
$$

for $\varphi \in \mathscr{D}\left(\mathbf{R}^{d_{1}}\right)$ and $\psi \in \mathscr{D}\left(\mathbf{R}^{d_{2}}\right)$.

By Proposition 2 and Proposition 3, we obtain

$$
\left\langle R_{0}, \varphi \otimes \psi\right\rangle=\langle k \psi, \varphi\rangle
$$

as $t \rightarrow+0$ for $\varphi \in \mathscr{S}\left(A_{1}\right)$ and $\psi \in \mathscr{S}\left(A_{2}\right)$.

\section{A Characterization of the Tempered Distributions Supported by a Regular Closed Set in the Heisenberg Group by the Heat Kernel Method}

\subsection{The Heisenberg Group $\mathbf{H}^{d}$}

We recall the definition and the properties of the Heisenberg group. We refer to [3], [5], [9], [19], [20], [23] and [24].

Let $g=(x, y, t)$ and $g^{\prime}=\left(x^{\prime}, y^{\prime}, t^{\prime}\right) \in \mathbf{R}^{d} \times \mathbf{R}^{d} \times \mathbf{R}=\mathbf{R}^{2 d+1}$. Then we define the group law of $\mathbf{R}^{2 d+1}$ by

$$
(x, y, t)\left(x^{\prime}, y^{\prime}, t^{\prime}\right)=\left(x+x^{\prime}, y+y^{\prime}, t+t^{\prime}+2\left(x^{\prime} \cdot y-x \cdot y^{\prime}\right)\right),
$$

where $x \cdot y=\sum_{j=1}^{d} x_{j} y_{j}$. The group $\mathbf{R}^{2 d+1}$ with respect to the group law defined by (3.1) is called the Heisenberg group and denoted by $\mathbf{H}^{d}$. Its identity element is $(0,0,0)$ and the inverse of the element $(x, y, t)$ is $(x, y, t)^{-1}=(-x,-y,-t)$. The Heisenberg group $\mathbf{H}^{d}$ is a locally compact Hausdorff group and its Haar measure is the Lebesgue measure $d x d y d t$.

The left-invariant vector fields in the Heisenberg group $\mathbf{H}^{d}$ as $\mathbf{R}^{2 d+1}$ are represented by

$$
X_{j}=\partial / \partial x_{j}+2 y_{j} \partial / \partial t, \quad X_{d+j}=\partial / \partial y_{j}-2 x_{j} \partial / \partial t \quad \text { and } \quad X_{2 d+1}=\partial / \partial t
$$

for $j=1,2, \ldots, d$ and these make a basis for the Lie algebra of $\mathbf{H}^{d}$. 
The sub-Laplacian $\Delta_{\mathbf{H}^{d}}$ on $\mathbf{H}^{d}$ is defined by $\Delta_{\mathbf{H}^{d}}=\sum_{j=1}^{2 d} X_{j}^{2}$. We consider the heat operator

on $\mathbf{H}^{d} \times(0, \infty)$.

$$
\partial / \partial s-\Delta_{\mathbf{H}^{d}}
$$

Let $\lambda>0$. Then we define the dilations $\delta_{\lambda}$ by

$$
\delta_{\lambda}(x, y, t)=\left(\lambda x, \lambda y, \lambda^{2} t\right)
$$

for $(x, y, t) \in \mathbf{H}^{d}$.

The homogeneous dimension $Q$ of $\mathbf{H}^{d}$ is given by

$$
Q=2 d+2 \text {. }
$$

Moreover, a function $u$ from $\mathbf{H}^{d}$ to $\mathbf{C}$ is called the Heisenberg-homogeneous of degree $k \in \mathbf{Z}$ if $u \circ \delta_{\lambda}=\lambda^{k} u$ for $\lambda>0$. Especially the Heisenberg-homogeneous of degree of the distance function $\rho$ defined by

$$
\rho(g)=\left(\left(x^{2}+y^{2}\right)^{2}+t^{2}\right)^{1 / 4}
$$

for $g \in \mathbf{H}^{d}$ is one, that is,

$$
\rho\left(\lambda x, \lambda y, \lambda^{2} t\right)=\lambda \rho(x, y, t) .
$$

The distance between two points $g$ and $g^{\prime}$ in $\mathbf{H}^{d}$ is given by

$$
d_{K}\left(g, g^{\prime}\right):=\rho\left(g^{\prime-1} g\right)
$$

and the following estimate holds:

$$
\rho\left(g^{\prime-1} g\right) \leq \rho(g)+\rho\left(g^{\prime}\right) .
$$

This distance function $\rho$ is called Korányi norm.

Let $f$ and $h$ be suitable functions on $\mathbf{H}^{d}$. Then we define the convolution $f * h$ of $f$ with $h$ as follows:

$$
(f * h)(g)=\int_{\mathbf{H}^{d}} f\left(g^{\prime}\right) h\left(g^{\prime-1} g\right) d g^{\prime}
$$

for $g, g^{\prime} \in \mathbf{H}^{d}$. The convolution on $\mathbf{H}^{d}$ is non-commutative, in general.

\subsection{The Space $\mathscr{S}\left(\mathbf{H}^{d}\right)$ and Its Dual Space $\mathscr{S}^{\prime}\left(\mathbf{H}^{d}\right)$}

Let $\alpha \in \mathbf{Z}_{+}^{2 d}$. Then the functions $\left(X_{\alpha} \varphi\right)(g)$ are defined by

$$
\left(X_{\alpha} \varphi\right)(g)=\left(X_{1}^{\alpha_{1}} X_{2}^{\alpha_{2}} \cdots X_{2 d}^{\alpha_{2 d}} \varphi\right)(g)
$$

for a function $\varphi \in C^{\infty}\left(\mathbf{H}^{d}\right)$. 
We define the Schwartz class $\mathscr{S}\left(\mathbf{H}^{d}\right)$ on the Heisenberg group as follows:

Definition 5. For any $\varphi \in C^{\infty}\left(\mathbf{H}^{d}\right)$, we say $\varphi \in \mathscr{S}\left(\mathbf{H}^{d}\right)$ if the function $\varphi$ satisfies the following condition: For any $N \in \mathbf{Z}_{+}$, we have

$$
\|\varphi\|_{N, \mathbf{H}^{d}}=\sup _{g \in \mathbf{H}^{d}}(1+\rho(g))^{N} \sum_{|\alpha| \leq N}\left|X_{\alpha} \varphi(g)\right|<\infty .
$$

It is clear from the definition that the space $\mathscr{S}\left(\mathbf{H}^{d}\right)$ is topologically isomorphic of the space $\mathscr{S}\left(\mathbf{R}^{2 d+1}\right)$. Moreover, it is known that the Schwartz class $\mathscr{S}\left(\mathbf{H}^{d}\right)$ is a Fréchet space in [3].

Definition 6. We denote by $\mathscr{S}^{\prime}\left(\mathbf{H}^{d}\right)$ the dual space of the space $\mathscr{S}\left(\mathbf{H}^{d}\right)$ and call it the space of the tempered distributions in the Heisenberg group. Thus, $u \in \mathscr{S}^{\prime}\left(\mathbf{H}^{d}\right)$ if and only if $u$ is a linear functional from $\mathscr{S}\left(\mathbf{H}^{d}\right)$ to $\mathbf{C}$ and satisfies the following condition: There exist $N \in \mathbf{Z}_{+}$and a positive constant $C$ such that

$$
|\langle u, \varphi\rangle| \leq C\|\varphi\|_{N, \mathbf{H}^{d}}
$$

for any $\varphi \in \mathscr{S}\left(\mathbf{H}^{d}\right)$.

By the definition, we can see that the space $\mathscr{S}^{\prime}\left(\mathbf{H}^{d}\right)$ is topologically isomorphic of the space $\mathscr{S}^{\prime}\left(\mathbf{R}^{2 d+1}\right)$.

Let $\check{f}(g)=f\left(g^{-1}\right)$ for $g \in \mathbf{H}^{d}$. Then we define the convolution $u * \varphi$ of $u \in \mathscr{S}^{\prime}\left(\mathbf{H}^{d}\right)$ with $\varphi \in \mathscr{S}\left(\mathbf{H}^{d}\right)$ as follows:

$$
\langle u * \varphi, \psi\rangle=\langle u, \psi * \check{\varphi}\rangle
$$

for any $\psi \in \mathscr{S}\left(\mathbf{H}^{d}\right)$.

\subsection{The Heat Kernel Method for the Space $\mathscr{S}^{\prime}\left(\mathbf{H}^{d}\right)$}

In [7] and [10], we can find the explicit form of the heat kernel (the fundamental solutions) $P_{s}(g)$ of the heat operator

on $\mathbf{H}^{d}$ as follows:

$$
\partial / \partial s-\Delta_{\mathbf{H}^{d}}
$$

$$
\begin{aligned}
P_{s}(g) & =P_{s}(x, y, t) \\
& =\left\{\begin{array}{l}
(4 \pi s)^{-(d+1)} \int_{-\infty}^{\infty}(2 \tau / \sinh 2 \tau)^{d} e^{i \tau t / 2 s-2\left(|x|^{2}+|y|^{2}\right) \tau /(4 s \tanh 2 \tau)} d \tau, \quad s>0 \\
0, \quad s \leq 0 .
\end{array}\right.
\end{aligned}
$$


The following properties of the heat kernel $P_{s}(g)$ hold:

Proposition $5([6])$. Let $P_{s}$ be the heat kernel associated to the sub-Laplacian $\Delta_{\mathbf{H}^{d}}$. Then the following properties hold:

(i) $P_{s}(g) \geq 0$,

(ii) $\int_{\mathbf{H}^{d}} P_{s}(g) d g=1$,

(iii) $P_{s}(g)=P_{s}\left(g^{-1}\right)$,

(iv) $\left(\partial / \partial s-\Delta_{\mathbf{H}^{d}}\right) P_{s}(g)=0$,

(v) $\lim _{s \rightarrow+0} P_{s}=\delta$ in $\mathscr{S}^{\prime}\left(\mathbf{H}^{d}\right)$,

(vi) $P_{r^{2} s}\left(r x, r y, r^{2} t\right)=r^{-Q} P_{s}(x, y, t), r>0,(x, y, t) \in \mathbf{H}^{d}$.

Moreover the heat kernel $P_{s}(g)$ has the following estimate:

Proposition 6 ([11]). Let $P_{s}(g)$ be the heat kernel associated to the subLaplacian $\Delta_{\mathbf{H}^{d}}$. Then for any $\alpha \in \mathbf{Z}_{+}^{2 d}$ and $m \in \mathbf{Z}_{+}$, there exist positive constants $a$ and $C_{m, \alpha}$ such that

$$
\left|(\partial / \partial s)^{m} X_{\alpha} P_{s}(g)\right| \leq C_{m, \alpha} s^{-m-|\alpha| / 2-Q / 2} e^{-a \rho(g)^{2} / s} .
$$

The following result is known:

Proposition 7 ([12], [17]). The heat kernel $P_{s}(g)$ is in the space $\mathscr{S}\left(\mathbf{H}^{d}\right)$ for $s>0$. Moreover for any $\varphi \in \mathscr{S}\left(\mathbf{H}^{d}\right)$, the following property holds:

$$
\varphi * P_{s} \rightarrow \varphi \in \mathscr{S}\left(\mathbf{H}^{d}\right)
$$

as $s$ converges to +0 .

J. Kim and M. W. Wong obtained the following characterization of the space $\mathscr{S}^{\prime}\left(\mathbf{H}^{d}\right)$. We call this characterization "the heat kernel method for $\mathscr{S}^{\prime}\left(\mathbf{H}^{d}\right)$ ":

THEOREM 4 ([12]). For $u \in \mathscr{S}^{\prime}\left(\mathbf{H}^{d}\right)$, we put

$$
U_{s}(g)=\left(u * P_{s}\right)(g)
$$

for $g \in \mathbf{H}^{d}$ and $s>0$. Then the function $U_{s}(g)$ satisfies the following four conditions:

(i) $U_{s}(g) \in C^{\infty}\left(\mathbf{H}^{d} \times(0, \infty)\right)$,

(ii) $\left(\partial / \partial s-\Delta_{\mathbf{H}^{d}}\right) U_{s}(g)=0, g \in \mathbf{H}^{d}$ and $s>0$, 
(iii) for any $\varphi \in \mathscr{S}\left(\mathbf{H}^{d}\right)$,

$$
\langle u, \varphi\rangle=\lim _{s \rightarrow+0} \int_{\mathbf{H}^{d}} U_{s}(g) \varphi(g) d g
$$

and

(iv) there exist $\mu, v>0$ and a constant $C>0$ such that

$$
\left|U_{s}(g)\right| \leq C s^{-\mu}(1+\rho(g))^{v}, \quad 0<s<1,
$$

for $g \in \mathbf{H}^{d}$.

Conversely every $U_{s}(g) \in C^{\infty}\left(\mathbf{H}^{d} \times(0, \infty)\right)$ satisfying the conditions (ii) and (iv) can be expressed in the form

$$
U_{s}(g)=\left(u * P_{s}\right)(g)
$$

with the unique element $u \in \mathscr{S}^{\prime}\left(\mathbf{H}^{d}\right)$.

\subsection{The Structure of the Tempered Distributions Supported by Regular Closed Sets in the Heisenberg Group}

At first, we give the definition of a regular closed set on $\mathbf{H}^{d}$.

Definition 7. Let $A_{\mathbf{H}^{d}}$ be a closed subset of the set $\mathbf{H}^{d}=\mathbf{R}^{2 d+1}$. If there exist $\kappa>0, \omega>0$ and $0<q \leq 1$ such that any $g_{1}$ and $g_{2} \in A_{\mathbf{H}^{d}}$ so that $\rho\left(g_{2}^{-1} g_{1}\right) \leq \kappa$ are linked by a curve in $A_{\mathbf{H}^{d}}$ whose length $l$ satisfies $l \leq$ $\omega \rho\left(g_{2}^{-1} g_{1}\right)^{q}$, then we call $A_{\mathbf{H}^{d}}$ a regular in the Heisenberg group $\mathbf{H}^{d}$.

We define the space $\mathscr{S}\left(A_{\mathbf{H}^{d}}\right)$ as follows:

Definition 8. Let $A_{\mathbf{H}^{d}}$ be a regular closed set on $\mathbf{H}^{d}$. For any $\varphi \in C^{\infty}\left(\mathbf{H}^{d}\right)$, we say $\varphi \in \mathscr{S}\left(A_{\mathbf{H}^{d}}\right)$ if the function $\varphi$ satisfies the following condition: For any $N \in \mathbf{Z}_{+}$, we have

$$
\|\varphi\|_{N, A_{\mathbf{H}^{d}}}=\sup _{g \in A_{\mathbf{H}^{d}}}(1+\rho(g))^{N} \sum_{|\alpha| \leq N}\left|X_{\alpha} \varphi(g)\right|<\infty
$$

The following relationship between the spaces $\mathscr{S}\left(\mathbf{H}^{d}\right)$ and $\mathscr{S}\left(A_{\mathbf{H}^{d}}\right)$ holds:

Proposition 8. The space $\mathscr{S}\left(\mathbf{H}^{d}\right)$ is dense in the space $\mathscr{S}\left(A_{\mathbf{H}^{d}}\right)$. 
Proof. It is enough that the space $\mathscr{D}\left(\mathbf{H}^{d}\right)$ is dense in the space $\mathscr{S}\left(A_{\mathbf{H}^{d}}\right)$. We choose $\Theta_{j} \in \mathscr{D}\left(\mathbf{H}^{d}\right)$ as follows:

$$
\Theta_{j}(g)= \begin{cases}1, & \rho(g) \leq j \\ 0, & \rho(g) \geq 2 j\end{cases}
$$

for $j=1,2, \ldots$ Let $f$ be in $\mathscr{S}\left(A_{\mathbf{H}^{d}}\right)$. If we set $\psi_{j}=f \Theta_{j}$, the function $\psi_{j}$ is in $\mathscr{D}\left(\mathbf{H}^{d}\right)$. On the other hand, we have

$$
X_{\alpha}\left\{\left(1-\Theta_{j}\right) f\right\}=\sum_{\beta \leq \alpha}\left(\begin{array}{l}
\alpha \\
\beta
\end{array}\right) X_{\beta}\left(1-\Theta_{j}\right) X_{\alpha-\beta} f .
$$

For $\rho(g) \leq j$, we can see

$$
X_{\beta}\left(1-\Theta_{j}\right)=0 .
$$

If the set $A_{\mathbf{H}^{d}}$ is compact, then by (3.3), we can see that

$$
\lim _{j \rightarrow \infty}\left\|f-\psi_{j}\right\|_{N, A_{\mathbf{H}^{d}}}=0 .
$$

On the other hand, for unbounded sets $A_{\mathbf{H}^{d}}$, we obtain the following estimate: For a sufficient large $j$, we have

$$
\begin{aligned}
\| f & -\psi_{j} \|_{N, A_{\mathbf{H}^{d}}} \\
& =\left\|\left(1-\Theta_{j}\right) f\right\|_{N, A_{\mathbf{H}^{d}}} \\
& \leq \sup _{g \in A_{\mathbf{H}^{d}} \backslash\left(\{\rho(g) \leq j\} \cap A_{\mathbf{H}^{d}}\right)}(1+\rho(g))^{N} \sum_{|\alpha| \leq N}\left|X_{\alpha}\left\{\left(1-\Theta_{j}\right) f\right\}\right| \\
& \leq \sup _{g \in A_{\mathbf{H}^{d}} \backslash\left(\{\rho(g) \leq j\} \cap A_{\mathbf{H}^{d}}\right)}(1+\rho(g))^{N} \sum_{|\alpha| \leq N}\left(\sum_{\beta \leq \alpha}\left(\begin{array}{c}
\alpha \\
\beta
\end{array}\right)\left|X_{\beta}\left(1-\Theta_{j}\right)\right|\left|X_{\alpha-\beta} f\right|\right) .
\end{aligned}
$$

Since $f \in \mathscr{S}\left(A_{\mathbf{H}^{d}}\right)$, we have for $\beta \leq \alpha(|\alpha| \leq N)$,

$$
(1+\rho(g))^{N}\left|X_{\alpha-\beta} f\right| \rightarrow 0
$$

as $j \rightarrow+\infty$. By (3.4), for any $f \in \mathscr{S}\left(A_{\mathbf{H}^{d}}\right)$, there exists the sequence $\left\{\psi_{j}\right\}_{j \in \mathbf{N}} \subset$ $\mathscr{D}\left(\mathbf{H}^{d}\right)$ such that

$$
\lim _{j \rightarrow+\infty}\left\|f-\psi_{j}\right\|_{N, A_{\mathbf{H}^{d}}}=0
$$

Therefore we can see that the space $\mathscr{D}\left(\mathbf{H}^{d}\right)$ is dense in the space $\mathscr{S}\left(A_{\mathbf{H}^{d}}\right)$. 
Definition 9. We denote by $\mathscr{S}\left(A_{\mathbf{H}^{d}}\right)^{\prime}$ the dual space of the space $\mathscr{S}\left(A_{\mathbf{H}^{d}}\right)$. Thus, $u \in \mathscr{S}\left(A_{\mathbf{H}^{d}}\right)^{\prime}$ if and only if $u$ is a linear functional from $\mathscr{S}\left(A_{\mathbf{H}^{d}}\right)$ to $\mathbf{C}$ and satisfies the following condition: There exist $N \in \mathbf{Z}_{+}$and a positive constant $C$ such that

$$
|\langle u, \varphi\rangle| \leq C\|\varphi\|_{N, A_{\mathbf{H}^{d}}}
$$

for any $\varphi \in \mathscr{S}\left(A_{\mathbf{H}^{d}}\right)$.

Here we denote by $\mathscr{S}_{A_{\mathbf{H}^{d}}}^{\prime}$ the space of the tempered distributions $u$ on $\mathbf{H}^{d}$ satisfying the following condition: For any $\varphi \in \mathscr{S}\left(\mathbf{H}^{d}\right)$, there exists a constant $C>0$ such that

$$
|\langle u, \varphi\rangle| \leq C\|\varphi\|_{N, A_{\mathbf{H}^{d}}}
$$

for some $N \in \mathbf{Z}_{+}$. We call the space $\mathscr{S}_{A_{\mathbf{H}^{d}}^{\prime}}^{\prime}$ as the space of the tempered distributions supported by $A_{\mathbf{H}^{d}}$ in $\mathbf{H}^{d}$. Then by Proposition 8, (3.5) means that $u$ has continuous on $\mathscr{S}^{\prime}\left(\mathbf{H}^{d}\right)$ with respect to the relative topology from $\mathscr{S}\left(A_{\mathbf{H}^{d}}\right)$. Hence $u$ has a unique linear continuous extension $u_{A_{\mathbf{H}^{d}}}$ on $\mathscr{S}\left(A_{\mathbf{H}^{d}}\right)$. This means that any tempered distributions with supported by $A_{\mathbf{H}^{d}}$ in $\mathbf{H}^{d}$ can be identified with an element of $\mathscr{S}\left(A_{\mathbf{H}^{d}}\right)^{\prime}$. Thus, we identify the space $\mathscr{S}_{A_{\mathbf{H}^{d}}^{\prime}}^{\prime}$ with the space $\mathscr{S}\left(A_{\mathbf{H}^{d}}\right)^{\prime}$.

Concerning on the element of $\mathscr{S}\left(A_{\mathbf{H}^{d}}\right)^{\prime}$, we obtain the following structure theorem (Bros-Epstein-Glaser type theorem).

Proposition 9. Let $A_{\mathbf{H}^{d}}$ be a regular closed set on $\mathbf{H}^{d}$. If $f \in \mathscr{S}\left(A_{\mathbf{H}^{d}}\right)^{\prime}$, then there exist the tempered measures supported on $A_{\mathbf{H}^{d}}, \mu_{\gamma}\left(|\gamma| \leq N, \gamma \in \mathbf{Z}_{+}^{d}\right)$, such that supp $\mu_{\gamma} \subset A_{\mathbf{H}^{d}}$ and

$$
f=\sum_{|\gamma| \leq N} X_{\gamma} \mu_{\gamma}
$$

where the tempered measure $\mu_{\gamma}$ means that there exists $N \in \mathbf{Z}_{+}$so that

$$
\int_{A_{\mathbf{H}^{d}}}\left|d \mu_{\gamma}\right|(g) /(1+\rho(g))^{N}<\infty .
$$

Proof. Let $f \in \mathscr{S}\left(A_{\mathbf{H}^{d}}\right)^{\prime}$. Thus, $f$ is in $\mathscr{S}^{\prime}\left(\mathbf{H}^{d}\right)$ with supp $f \subset A_{\mathbf{H}^{d}}$, where $A_{\mathbf{H}^{d}}$ is a regular closed set in $\mathbf{H}^{d}$. Then we can see that for any $\varphi \in \mathscr{S}\left(\mathbf{H}^{d}\right)$, there exists a constant $C>0$ such that

$$
|\langle f, \varphi\rangle| \leq C\|\varphi\|_{N, A_{\mathbf{H}^{d}}} .
$$


On the other hand, let $\Phi_{N, A_{\mathbf{H}^{d}}}$ be the set spanned by all finite linear combinations of the bounded continuous functions on $A_{\mathbf{H}^{d}},\left\{\varphi_{\gamma},|\gamma| \leq N\right\}$, and we define the norm as follows:

$$
\left\|\left\{\varphi_{\gamma}\right\}\right\|_{\mathbf{H}^{d}}=\sup _{g \in A_{\mathbf{H}^{d}},|\gamma| \leq N}\left|\varphi_{\gamma}(g)\right| .
$$

Let the space $\Psi$ be spanned by all finite linear combinations of

$$
\left\{\varphi_{\gamma}(g)=(1+\rho(g))^{N} \sum_{|\gamma| \leq N} X_{\gamma} \varphi(g), \varphi \in \mathscr{S}\left(\mathbf{H}^{d}\right)\right\}
$$

as a subspace of $\Phi_{N, A_{\mathbf{H}^{d}}}$. If we define the bounded linear functional $\tilde{f}$ on $\Psi$ by

$$
\left\langle\tilde{f}, \varphi_{\gamma}\right\rangle=\langle f, \varphi\rangle
$$

then by Hahn-Banach theorem, we can extend $\tilde{f}$ on $\Phi_{N, A_{\mathbf{H}^{d}}}$ and there exists a constant $C>0$ such that

$$
\left|\left\langle\tilde{f}, \varphi_{\gamma}\right\rangle\right| \leq C\left\|\left\{\varphi_{\gamma}\right\}\right\|_{\mathbf{H}^{d}} .
$$

Therefore there exists the measure $v_{\gamma}$ on $A_{\mathbf{H}^{d}}$ such that

$$
v_{\gamma}: \int_{A_{\mathbf{H}^{d}}}\left|d v_{\gamma}(g)\right|<\infty
$$

and we can express $\left\langle\tilde{f}, \varphi_{\gamma}\right\rangle$ by

$$
\left\langle\tilde{f}, \varphi_{\gamma}\right\rangle=\int_{A_{\mathbf{H}^{d}}} \varphi_{\gamma}(g) d v_{\gamma}(g) .
$$

Hence by (3.6), we have

$$
\langle f, \varphi\rangle=\sum_{|\gamma| \leq N} \int_{A_{\mathbf{H}^{d}}}(1+\rho(g))^{N} X_{\gamma} \varphi(g) d v_{\gamma}(g), \quad \varphi \in \mathscr{S}\left(\mathbf{H}^{d}\right) .
$$

If we put $\tilde{\boldsymbol{v}}_{\gamma}\left(\mathbf{H}^{d}\right)=v_{\gamma}\left(A_{\mathbf{H}^{d}} \cap \mathbf{H}^{d}\right)$ and $\mu_{\gamma}=(1+\rho(g))^{N} \tilde{\boldsymbol{v}}_{\gamma}$, then the measure $\mu_{\gamma}$ extended on $\mathbf{H}^{d}$ is the tempered measure supported by $A_{\mathbf{H}^{d}}$. Finally, we obtain

$$
f=\sum_{|\gamma| \leq N} X_{\gamma} \mu_{\gamma}
$$

\subsection{The Heat Kernel Method for the Space of the Tempered Distributions Supported by a Regular Closed Set on $\mathbf{H}^{d}$}

Our main result is as follows: 
THEOREM 5. Let $A$ be a regular closed set on $\mathbf{H}^{d}$. For any $u$ in $\mathscr{S}\left(A_{\mathbf{H}^{d}}\right)^{\prime}$, let $U_{s}(g)=\left\langle u, P_{s}\left(\cdot{ }^{-1} g\right)\right\rangle$. Then $U_{s}(g)$ satisfies the following conditions:

(i) $U_{s}(g) \in C^{\infty}\left(\mathbf{H}^{d} \times(0, \infty)\right)$,

(ii) $\left(\partial / \partial s-\Delta_{\mathbf{H}^{d}}\right) U_{s}(g)=0, g \in \mathbf{H}^{d}$ and $s>0$,

(iii) for any $\varphi \in \mathscr{D}\left(\mathbf{H}^{d}\right)$,

$$
\langle u, \varphi\rangle=\lim _{s \rightarrow+0} \int_{\mathbf{H}^{d}} U_{s}(g) \varphi(g) d g
$$

and

(iv) there exist $\mu, v>0$ and constants $C>0$ and a such that

$$
\left|U_{s}(g)\right| \leq C s^{-\mu}(1+\rho(g))^{v} e^{-a \rho\left(g, A_{\mathbf{H}^{d}}\right)^{2} / 2 s}, \quad 0<s<1,
$$

for $g \in \mathbf{H}^{d}$, where $\rho\left(g, A_{\mathbf{H}^{d}}\right)=\inf _{g^{\prime} \in A_{\mathbf{H}^{d}}} \rho\left(g^{\prime-1} g\right)$.

Conversely every $U_{s}(g) \in C^{\infty}\left(\mathbf{H}^{d} \times(0, \infty)\right)$ satisfying the conditions (ii) and (iv) can be expressed in the form

$$
U_{s}(g)=\left(u * P_{s}\right)(g)
$$

with the unique element $u \in \mathscr{S}\left(A_{\mathbf{H}^{d}}\right)^{\prime}$.

Proof. Let $u \in \mathscr{S}\left(A_{\mathbf{H}^{d}}\right)^{\prime}$. If we set

$$
U_{s}(g)=\left\langle u, P_{s}\left(\cdot \cdot^{-1} g\right)\right\rangle,
$$

then by Proposition 6, Proposition 9 and (3.2), there exist positive constants $a$ and $C$ such that

$$
\begin{aligned}
\left|U_{s}(g)\right| & =\left|\left\langle u, P_{s}\left(\cdot^{-1} g\right)\right\rangle\right| \\
& =\left|\sum_{|\gamma| \leq N} \int_{A_{\mathbf{H}^{d}}}(1+\rho(g))^{N} X_{\gamma} P_{s}\left(g_{1}^{-1} g\right) d \mu_{\gamma}\left(g_{1}\right)\right| \\
& \leq \sum_{|\gamma| \leq N} \int_{A_{\mathbf{H}^{d}}}\left(1+\rho\left(g_{1}\right)\right)^{N}\left|X_{\gamma} P_{s}\left(g_{1}^{-1} g\right)\right|\left|d \mu_{\gamma}\right|\left(g_{1}\right) \\
& \leq C_{\gamma} s^{-|\gamma| / 2-Q / 2} \int_{A_{\mathbf{H}^{d}}}\left(1+\rho\left(g_{1}\right)\right)^{N} e^{-a \rho\left(g_{1}^{-1} g\right)^{2} / s}\left|d \mu_{\gamma}\right|\left(g_{1}\right) \\
& \leq C_{\gamma} S^{-|\gamma| / 2-Q / 2}(1+\rho(g))^{N} \int_{A_{\mathbf{H}^{d}}}\left(1+\rho\left(g_{1}^{-1} g\right)\right)^{N} e^{-a \rho\left(g_{1}^{-1} g\right)^{2} / s}\left|d \mu_{\gamma}\right|\left(g_{1}\right) \\
& \leq C_{\gamma, N} s^{-|\gamma| / 2-Q / 2}(1+\rho(g))^{N} e^{-a \rho\left(g, A_{\mathbf{H}^{d}}\right)^{2} / 2 s} .
\end{aligned}
$$


Conversely, for any $U_{s}(g) \in C^{\infty}\left(\mathbf{H}^{d} \times(0, \infty)\right)$ satisfying the assumption (ii) and (iv) in Theorem 5, by Theorem 4, there exists $u \in \mathscr{S}^{\prime}\left(\mathbf{H}^{d}\right)$ such that

$$
U_{s}(g)=\left\langle u, P_{s}\left(\cdot{ }^{-1} g\right)\right\rangle .
$$

Let $\varphi \in \mathscr{D}\left(\mathbf{H}^{d}\right)$ and $K=\operatorname{supp} \varphi \subset \mathbf{H}^{d} \backslash A_{\mathbf{H}^{d}}$. Then there are a constant $C_{K, v}>0$ and $\mu \geq 0$ such that

$$
\begin{aligned}
\left|\int_{K} U_{s}(g) \varphi(g) d g\right| & \leq \int_{K}\left|U_{s}(g)\right||\varphi(g)| d g \\
& \leq C_{K, v} s^{-\mu} e^{-a \rho\left(K, A_{\left.\mathbf{H}^{d}\right)^{2} / 2 s}\right.} \\
& \rightarrow 0,
\end{aligned}
$$

as $s \rightarrow+0$, where $\rho\left(K, A_{\mathbf{H}^{d}}\right)=\inf _{g \in K} \rho\left(g, A_{\mathbf{H}^{d}}\right)$. Hence we obtain

$$
\lim _{s \rightarrow+0} \int_{\mathbf{H}^{d}} U_{s}(g) \varphi(g) d g=0 .
$$

On the other hand, by Theorem 4, we have

$$
\lim _{s \rightarrow+0} U_{s}(g)=u(g) \text { in } \mathscr{S}^{\prime}\left(\mathbf{H}^{d}\right) .
$$

Therefore we can see that $\operatorname{supp} u \subset A_{\mathbf{H}^{d}}$.

\subsection{Schwartz Kernel Theorem for the Space $\mathscr{S}\left(A_{\mathbf{H}^{d}}\right)^{\prime}$}

As an application of the characterization of the space $\mathscr{S}\left(A_{\mathbf{H}^{d}}\right)^{\prime}$, we show the Schwartz kernel theorem for the space $\mathscr{S}\left(A_{\mathbf{H}^{d}}\right)^{\prime}$ as follows:

THEOREM 6. Let the sets $A_{\mathbf{H}^{d_{1}}}$ and $A_{\mathbf{H}^{d_{2}}}$ be regular closed sets on $\mathbf{H}^{d_{1}}$ and $\mathbf{H}^{d_{2}}$ respectively and $k$ be a continuous linear operator from $\mathscr{S}\left(A_{\mathbf{H}^{d_{2}}}\right)$ to $\mathscr{S}\left(A_{\mathbf{H}^{d_{1}}}\right)^{\prime}$. Then there exists $T$ in $\mathscr{S}\left(A_{\mathbf{H}^{d_{1}}} \times A_{\mathbf{H}^{d_{2}}}\right)^{\prime}$ such that

$$
\langle k \psi, \varphi\rangle=\langle T, \varphi \otimes \psi\rangle,
$$

where $\varphi$ is in $\mathscr{S}\left(A_{\mathbf{H}^{d_{1}}}\right)$ and $\psi$ is in $\mathscr{S}\left(A_{\mathbf{H}^{d_{2}}}\right)$.

Proof. Since $k$ is continuous, the bilinear form $\mathbf{B}$ on $\mathscr{S}\left(A_{\mathbf{H}^{d_{1}}}\right) \times \mathscr{S}\left(A_{\mathbf{H}^{d_{2}}}\right)$,

$$
\mathbf{B}(\varphi, \psi)=\langle k \psi, \varphi\rangle, \quad \varphi \in \mathscr{S}\left(A_{\mathbf{H}^{d_{1}}}\right), \psi \in \mathscr{S}\left(A_{\mathbf{H}^{d_{2}}}\right)
$$

is separately continuous. Since $\mathscr{S}\left(A_{\mathbf{H}^{d_{1}}}\right)$ and $\mathscr{S}\left(A_{\mathbf{H}^{d_{2}}}\right)$ are the Fréchet space respectively, $\mathbf{B}$ is continuous. Hence we can see that there exist a positive constant 
$C$ and $N_{1}, N_{2} \in \mathbf{Z}_{+}$such that

$$
|\langle k \psi, \varphi\rangle| \leq C\|\varphi\|_{N_{1}, A_{\mathbf{H}^{d_{1}}}}\|\psi\|_{N_{2}, A_{\mathbf{H}^{d_{2}}}} .
$$

We define $R_{s}\left(g_{1}, g_{2}\right)$ by

$$
R_{s}\left(g_{1}, g_{2}\right)=\left\langle k P_{s}\left(g_{2}^{-1} \cdot\right), P_{s}\left(g_{1}^{-1} \cdot\right)\right\rangle
$$

for $\left(g_{1}, g_{2}\right) \in \mathbf{H}^{d_{1}} \times \mathbf{H}^{d_{2}}$ and $s>0$.

Now we will show $R_{s}$ converges in $\mathscr{S}\left(A_{\mathbf{H}^{d_{1}}} \times A_{\mathbf{H}^{d_{2}}}\right)^{\prime}$ as $s \rightarrow+0$. By (\#\#) and Proposition 6, there exist positive constants $a, C$ and $M, N_{1}, N_{2} \in \mathbf{Z}_{+}$such that

$$
\left|R_{s}\left(g_{1}, g_{2}\right)\right| \leq C s^{-M}\left(1+\rho\left(g_{1}\right)\right)^{N_{1}}\left(1+\rho\left(g_{2}\right)\right)^{N_{2}} e^{-a \rho\left(g_{1}, A_{\mathbf{H}^{d_{1}}}\right)^{2} / 2 s} e^{-a \rho\left(g_{2}, A_{\mathbf{H}^{d_{2}}}\right)^{2} / 2 s},
$$

for $g_{1} \in \mathbf{H}^{d_{1}}, g_{2} \in \mathbf{H}^{d_{2}}$ and $0<s<1$.

Moreover we obtain

$$
\left(\partial / \partial s-\Delta_{\mathbf{H}^{d_{1}+d_{2}}}\right) R_{s}\left(g_{1}, g_{2}\right)=0
$$

for $g_{1} \in \mathbf{H}^{d_{1}}, g_{2} \in \mathbf{H}^{d_{2}}$ and $0<s<1$.

Therefore, by Theorem 5, there exists $R_{0} \in \mathscr{S}\left(\mathbf{H}^{d_{1}} \times \mathbf{H}^{d_{2}}\right)^{\prime}, \quad$ supp $R_{0} \subset$ $A_{\mathbf{H}^{d_{1}}} \times A_{\mathbf{H}^{d_{2}}}$, such that

$$
R_{0}=\lim _{s \rightarrow+0} R_{s}
$$

in $\mathscr{S}^{\prime}\left(\mathbf{H}^{d_{1}} \times \mathbf{H}^{d_{2}}\right)$.

Since the Riemann sum of an integral converges in $\mathscr{S}\left(\mathbf{H}^{d_{j}}\right), j=1,2$, by Proposition 5, we have

$$
\begin{aligned}
\left\langle R_{s}, \varphi \otimes \psi\right\rangle & =\iint_{\mathbf{H}^{d_{1}} \times \mathbf{H}^{d_{2}}} R_{s}\left(g_{1}, g_{2}\right) \varphi\left(g_{1}\right) \psi\left(g_{2}\right) d g_{1} d g_{2} \\
& =\left\langle k \int_{\mathbf{H}^{d_{2}}} P_{s}\left(g_{2}^{-1} \cdot\right) \psi\left(g_{2}\right) d g_{2}, \int_{\mathbf{H}^{d_{1}}} P_{s}\left(g_{1}^{-1} \cdot\right) \varphi\left(g_{1}\right) d g_{1}\right\rangle \\
& =\left\langle k\left[\psi * P_{s}\right], \varphi * P_{s}\right\rangle
\end{aligned}
$$

for $\varphi \in \mathscr{D}\left(\mathbf{H}^{d_{1}}\right)$ and $\psi \in \mathscr{D}\left(\mathbf{H}^{d_{2}}\right)$.

Therefore by the argument of the density and Proposition 8, we obtain

$$
\left\langle R_{0}, \varphi \otimes \psi\right\rangle=\langle k \psi, \varphi\rangle,
$$

as $s \rightarrow+0$ for $\varphi \in \mathscr{S}\left(A_{\mathbf{H}^{d_{1}}}\right)$ and $\psi \in \mathscr{S}\left(A_{\mathbf{H}^{d_{2}}}\right)$. 


\section{Appendix: Some Example of a Regular Closed Set in the Heisenberg Group $\mathbf{H}$}

We recall the definition and the properties of geodesics and Geodetically convex sets in the Heisenberg group H. We refer to [2] and [16]. A curve $c=$ $(x, y, t) \in \mathbf{H} \cong \mathbf{R}^{3}$ is said to be horizontal if

$$
\dot{c} \in \operatorname{span}\{X, Y\}, \quad \dot{c}=\dot{x} X+\dot{y} Y .
$$

Any two points $P$ and $Q$ in $\mathbf{H}$ can be joined by horizontal (smooth) curve, that is, there exists a horizontal curve $c$ such that $c(0)=P, c(1)=Q$. The length of a horizontal curve $c$ is

$$
l(c)=\int_{0}^{1} \sqrt{g(\dot{c}(s), \dot{c}(s))} d s,
$$

where $g$ is the subRiemannian metric. Moreover the Carnot-Carathéodory distance $d_{C}$ is defined by

$d_{C}(P, Q)=\inf \{l(c), c$ are horizontal curves such that $c(0)=P, c(1)=Q\}$.

The Carnot-Carathéodory distance $d_{C}$ is a metric on $\mathbf{H}$ is bi-Lipschitz equivalent to Korányi distance $d_{K}$, that is, there a constant $C>1$ such that

$$
C^{-1} d_{K} \leq d_{C} \leq C d_{K}
$$

Definition 10 ([16]). A geodesic connecting two points $P, Q \in \mathbf{H}$ is a length minimizing horizontal curve $c$, that is, a curve $c$ such that $c(0)=P, c(T)=Q$ and $l(c)=d_{C}(P, Q)$.

Concerning on a geodesic, it is known that the following result holds:

Proposition 10 ([2]). Given a point $P(x, y, 0)$, there is a unique geodesics between the origin and $P$. It is a straight line in the plane $\{t=0\}$.

By Proposition 10, we obviously have the following example 1:

ExAmple 1. The line through the origin on the plane $\{t=0\}$ in $\mathbf{H}$ is a regular closed set in the Heisenberg group.

In [16], R. Monti and M. Rickly defined the geodetically convex sets in the Heisenberg group as follows: 
Definition 11 ([16]). We say that a set $C \subseteq \mathbf{H}$ is geodetically convex if for all $p_{0}, p_{1} \in C$ and all geodesics $c:[0, l] \rightarrow \mathbf{H}$ with $l=d\left(p_{0}, p_{1}\right), c(0)=p_{0}$ and $c(l)=p_{1}$, we have $c([0, l]) \subseteq C$.

Unfortunately, the family of geodetically convex sets is very poor differently from the Euclidean space as follows:

Proposition 11 ([16]). The only geodetically convex subsets of $\mathbf{H}$ are the empty set, points, arcs of geodesics and $\mathbf{H}$.

By Proposition 11, we have the following example:

EXAmple 2. The arcs of geodesics are a regular closed set in the Heisenberg group.

\section{References}

[ 1 ] R. Ashino, T. Mandai and A. Morimoto, System identification based on distribution theory and wavelet transform, Applicable Anal., Vol. 84, No. 2, February (2005), 165-195.

[2] O. Calin, D.-C. Chang and P. Greiner, Geometric Analysis in the Heisenberg Group and Its Generalizations, AMS/IP, (2007).

[3] L. Corwin and F. P. Greenleaf, Representations of Nilpotent Lie Groups and Their Applications: Volume 1, Part 1, Basic Theory and Examples, Cambridge University Press, Cambridge, (1990).

[4] C. Dong and T. Matsuzawa, $\mathscr{S}$-space of Gel'fand-Shilov and differential equations, Japan. J. Math. Vol. 19, No. 2 (1994), 227-239.

[ 5 ] G. B. Folland, Harmonic Analysis in Phase Space, Princeton University Press. Princeton, N.J., (1989).

[6] G. B. Folland, Subelliptic estimates and function spaces on nilpotent Lie group, Ark. Mat. 13 (1975), 161-207.

[ 7 ] B. Gaveau, Principe de moindre action, propagation de la chaleur, et estimées sous elliptiques sur certains groupes nilpotents, Acta Math. 139 (1977), 95-153.

[ 8 ] L. Hörmander, The Analysis of Linear Partial Differential Operators I, Springer-Verlag, Berlin, Heidelberg, New York (1983).

[9] R. Howe, On the role of the Heisenberg group in harmonic analysis, Bull. Amer. Math. Soc. 3 (1980), 821-843.

[10] A. Hulanicki, The distribution of energy in the Brownian motion in the Gaussian field and analytic hypoellipticity of certain subelliptic operators in the Heisenberg group, Studia Math. 56 (1976), 165-173.

[11] D. S. Jerison and A. Sánchez-Calle, Estimates for the heat kernel for a sum of squares of vector fields, Indiana Univ. Math. J., 35 (1986), 835-854.

[12] J. Kim and M. W. Wong, Positive definite temperature functions in the Heisenberg group, Appl. Anal. 85, No. 8, (2006), 987-1000.

[13] S. Lee and S.-Y. Chung, The Paley-Wiener theorem by the heat kernel method, Bull. Korean Math. Soc. 35, No. 3, (1998), 441-453.

[14] T. Matsuzawa, A calculus approach to the hyperfunctions I, Nagoya Math. J., 108 (1987), 53-66. 
[15] T. Matsuzawa, A calculus approach to the hyperfunctions III, Nagoya Math. J., 118 (1990), 133-153.

[16] R. Monti and M. Rickly, Geodetically convex sets in the Heisenberg group, J. Convex Anal., vol. 12, No. 1, (2005), 187-196.

[17] Y. Oka, The Schwartz kernel theorem for the tempered distributions in the Heisenberg group, to appear in Hokkaido Math. J..

[18] L. Schwartz, Théorie des distributions, Hermann, Paris, (1966).

[19] E. M. Stein, Harmonic Analysis: Real-Variable Methods, Orthogonality, and Oscillatory Integrals, Princeton University Press, Princeton, N. J., (1993).

[20] S. Thangavelu, An Introduction to the Uncertainty Principle: Hardy's Theorem on Lie Groups, Birkhäuser, Boston, (2004).

[21] V. S. Vladimirov, Les Fonctions de Plusieurs Variables Complexes, Dunod Paris, (1967).

[22] H. Whitney, Functions differentiable on the boundaries of regions, Ann. of Math., 35 (3), (1934), $482-485$.

[23] M. W. Wong, Weyl Transforms, Springer-Verlag, New York, (1998).

[24] M. W. Wong, Partial Differential Equations, Topics in Fourier Analysis CRC Press, Boca Raton (2014).

\author{
General Education \\ National Institute of Technology \\ Kushiro College \\ 2-32-1 Otanoshike-Nishi, Kushiro-Shi \\ Hokkaido 084-0916, Japan \\ E-mail: oka@kushiro-ct.ac.jp
}

